\title{
Evaluation of microRNA expression in plasma and skeletal muscle of thoroughbred racehorses in training
}

\author{
B. A. McGivney ${ }^{1}$, M. E. Griffin², K. F. Gough', C. L. McGivney', J. A. Browne ${ }^{1}$, E. W. Hill' ${ }^{1}$ and L. M. Katz²
}

\begin{abstract}
Background: Circulating miRNAs (ci-miRNAs) are endogenous, non-coding RNAs emerging as potential diagnostic biomarkers. Equine miRNAs have been previously identified including subsets of tissue-specific miRNAs. In order to investigate ci-miRNAs as diagnostic tools, normal patterns of expression for different scenarios including responses to exercise need to be identified. Human studies have demonstrated that many ci-miRNAs are up-regulated following exercise with changes in expression patterns in skeletal muscle. However, technical challenges such as haemolysis impact on accurate plasma ci-miRNA quantification, with haemolysis often occurring naturally in horses following moderateto-intense exercise. The objectives of this study were to identify plasma ci-miRNA profiles and skeletal muscle miRNAs before and after exercise in Thoroughbreds (Tb), and to evaluate for the presence and effect of haemolysis on plasma ci-miRNA determination. Resting and post-exercise plasma ci-miRNA profiles and haemolysis were evaluated in twenty 3 year-old Tbs in sprint training. Resting and post-exercise skeletal muscle miRNA abundance was evaluated in a second cohort of eleven 2 year-old Tbs just entering sprint training. Haemolysis was further quantified in resting blood samples from twelve Tbs in sprint training. A human plasma panel containing 179 miRNAs was used for profiling, with haemolysis assessed spectrophotometrically. Data was analysed using a paired Student's t-test and Pearson's rank correlation.

Results: Plasma ci-miRNA data for 13/20 horses and all skeletal muscle miRNA data passed quality control. From plasma, 52/179 miRNAs were detected at both time-points. Haemolysis levels were greater than the threshold for accurate quantification of ci-miRNAs in 18/25 resting and all post-exercise plasma samples. Positive correlations $(P<0.05)$ between haemolysis and miRNA abundance were detected for all but 4 miRNAs, so exercise-induced changes in plasma ci-miRNA expression could not be quantified. In skeletal muscle samples, 97/179 miRNAs were detected with 5 miRNAs (miR-21-5p, let-7d-3p, let-7d-5p, miR-30b-5p, miR-30e-5p) differentially expressed $(\mathrm{DE}, P<0.05)$ between time-points.

Conclusions: The degree of haemolysis needs to be determined prior to quantifying plasma ci-miRNA expression from horses in high-intensity exercise training. Identification of DE miRNAs in skeletal muscle indicates modification of miRNA expression may contribute to adaptive training responses in Tbs. Using a human plasma panel likely limited detection of equine-specific miRNAs.
\end{abstract}

Keywords: Horse, miRNA, Exercise, Plasma, Haemolysis, Skeletal muscle

\footnotetext{
*Correspondence: lisa.katz@ucd.ie

${ }^{2} U C D$ School of Veterinary Medicine, Section of Veterinary Biomedical

Sciences, University College Dublin, Belfield, Dublin 4, Ireland

Full list of author information is available at the end of the article
} 


\section{Background}

MicroRNAs (miRNAs) are endogenous, non-coding RNAs with complex roles causing translational repression/degradation of bound mRNA [1], regulating many cellular processes [2]. They have intracellular effects, are extremely stable when secreted into the bloodstream [3] and have been identified in multiple body fluids [4]. MiRNAs are often tissue-specific and can be secreted from their tissue of origin into the bloodstream bound to lipoproteins [5] or packaged into extracellular microparticles [6]. When in blood they are termed circulating miRNAs (ci-miRNAs) [7] and are emerging as potential diagnostic biomarkers due to their stability [8] and ease of access for sample collection.

Human studies have identified a multitude of cimiRNAs up-regulated following intense exercise training [9-12]. Furthermore, exercise training has been found to alter the expression pattern of miRNAs in skeletal muscle [11, 13, 14]. It has been hypothesised that skeletal muscle may contribute to changes in differentially expressed (DE) ci-miRNAs in response to exercise training by releasing exosomes into plasma $[15,16]$, supporting the possibility that DE plasma ci-miRNAs could be used to assess adaptive training responses of skeletal muscle.

MiRNA analysis in horses is an emerging area of research. To-date, $>700$ equine miRNAs have been identified with subsets of tissue-specific DE miRNAs isolated [17] including skeletal muscle and blood [18, 19]. Recently, 197 miRNAs were identified in equine skeletal muscle, with 76 found to be muscle-specific [20]. However, to-date there has only been one study evaluating ci-MRNA in exercising horses [21]. In this report, $167 \mathrm{DE}$ miRNAs were identified from pre- and post-endurance exercise blood samples; however, whole-blood rather than plasma/serum was evaluated making it difficult to determine if ci-miRNAs were expressed from red blood cells $(\mathrm{RBC})$ or other tissue sites such as skeletal muscle.

There are technical challenges involved in plasma/serum ci-miRNA measurement such as inaccurately quantifying plasma RNA due to low yields, haemolysis impacting on ci-miRNA quantification and a lack of validated stable reference miRNAs [22-25]. Since haemolysis may occur naturally in horses following moderate-to-intense exercise $[26,27]$, this needs to be considered when attempting to evaluate equine plasma/serum ci-miRNA expression.

The aim was to identify DE plasma ci-miRNAs and skeletal muscle miRNAs before and after exercise in Thoroughbreds $(\mathrm{Tb})$, and to evaluate for the presence and effect of haemolysis on plasma ci-miRNA determination.

\section{Methods}

A Department of Health License (B100/3525), ethical approval from the University College Dublin Animal
Research Ethics Committee and owner consent were obtained for all horses and procedures in this study.

\section{Sample population}

All horses in the study were from the same stable and were managed and trained in a similar way [28]. Horses trained 6 days per week with gradual introduction of 800-1000 m sprint training (work day [WD]) alternating with submaximal training, following which horses entered competitive racing.

\section{Experimental protocol \\ Cohort A: Plasma ci-miRNA}

Plasma lactate concentrations ([LA]) and ci-miRNA were evaluated in $n=20$ sex- and fitness-matched 3 year-old Tbs ( $n=10$ males, $n=10$ females) in active training and racing before $\left(\mathrm{T}_{0}\right)$ and 5 mins after $\left(\mathrm{T}_{5 \min }\right)$ a WD training session. The degree of haemolysis was evaluated in all samples.

\section{Cohort B: Skeletal muscle miRNA}

Resting $\left(\mathrm{T}_{0}\right)$ and $\mathrm{T}_{5 \min }$ plasma [LA] along with $\mathrm{T}_{0}$ and $4 \mathrm{~h}\left(\mathrm{~T}_{4 \mathrm{hr}}\right)$ post-exercise skeletal muscle miRNAs were evaluated in $n=112$ year-old Tbs $(n=7$ males, $n=4$ females) before and after their first WD training session.

\section{Cohort C: Degree of plasma haemolysis}

The degree of haemolysis was further evaluated in $\mathrm{T}_{0}$ plasma samples from an additional $n=12$ Tbs in active training and racing ( $n=5$ males, $n=7$ females, 2-4 years old).

\section{Data collection}

Prior to an exercise test horses were fitted with a heart rate (HR) telemetry system ${ }^{1}$ and GPS unit $^{2}$ which recorded speed, HR and distance.

Resting jugular venous blood samples were collected before feeding and exercise. Samples for plasma [LA] and ci-miRNA measurement were placed into fluoride oxalate and ethylenediamine tetraacetic acid (EDTA) tubes, respectively. Once collected all blood tubes were immediately centrifuged with plasma separated. [LA] was measured on-site with an autoanalyser. ${ }^{3}$ Plasma for haemoglobin concentrations $[\mathrm{Hb}]$ and ci-miRNA analysis was stored at $4{ }^{\circ} \mathrm{C}$ until transported to be stored at $-80{ }^{\circ} \mathrm{C}$ (within $6 \mathrm{~h}$ of collection) and analysed as a batch at a later date.

\section{Blood samples for miRNA}

MiRNAs were extracted from plasma samples ${ }^{4}$ with $4 \mu \mathrm{L}$ amounts then reversely transcribed in $20 \mu \mathrm{L}$ reactions. ${ }^{5}$ Exogenous RNA spike-ins (UniSp2, UniSp4, UniSp5, cel-miR-39-3p) provided by the manufacturer of the human serum/plasma focus miRNA PCR panel kit 
were added during RNA extraction and analysed according to the manufacturer's instructions ${ }^{5}$.

\section{Haemolysis analysis}

The degree of haemolysis was assessed by quantifying plasma $[\mathrm{Hb}]$ using a UV/VIS spectrophotometer, ${ }^{6}$ measuring oxyhaemoglobin absorbance at $\mathrm{k}=414 \mathrm{~nm}$ [22]. A value of $\leq 0.2$ absorption units (AU) was used as the threshold for acceptable haemolysis and accurate quantification of ci-miRNA detection [22].

\section{Skeletal muscle biopsy samples for miRNA}

Skeletal muscle biopsies were obtained from the gluteus medius using a $6 \mathrm{~mm}$-diameter, modified Bergstrom biopsy needle ${ }^{7}$ according to previously described methods [29]. Samples were preserved in RNAlater ${ }^{8}$ until further processing.

Total RNA was extracted from 50 to $100 \mathrm{mg}$ of tissue using a protocol combining TRIzol reagent, ${ }^{9}$ DNase treatment ${ }^{10}$ and RNeasy. ${ }^{11}$ RNA was quantified using a Qubit fluorimeter with the Qubit RNA HS assay. RNA quality was assessed using the $18 \mathrm{~S} / 28 \mathrm{~S}$ ratio and RNA integrity number on an Agilent Bioanalyzer with the RNA 6000 Nano Lab-Chip kit. ${ }^{12}$ RNA (2 $\mu$ L@5 ng/ul) was reversely transcribed in $10 \mu \mathrm{L}$ reactions ${ }^{5}$. UniSp6 (exogenous RNA spike-in) was added during cDNA conversion.

\section{Real-time quantitative polymerase chain reaction measurements}

cDNA was assayed in $10 \mu \mathrm{L}$ PCR reactions ${ }^{5}$. All miRNAs were assayed once by RT-qPCR on the Exiqon Human Serum/Plasma Focus miRNA PCR panel I and II. Each panel consisted of a 96 well plate with $179 \mathrm{miR}$ NAs of interest and 15 controls (inter-plate calibrators, negative and spike-in controls). Amplification was performed in an Applied Biosystems StepOnePlus ${ }^{\mathrm{Tn}}$ RealTime PCR System. Amplification curves were analysed using Applied Biosystems software to obtain raw PCR cycle threshold $\left(C_{\mathrm{t}}\right)$ values.

\section{miRNA quantification}

Quality control measures (inter-plate calibration, ommission of outliers, miRNAs with $C_{\mathrm{t}}$ values $>34$ from analysis) were carried out using GeneEx software. MiRNAs with $>80 \%$ missing data were removed from the analysis, with missing values replaced with the mean value for that miRNA. Results from muscle biopsies were normalised to the global mean.

\section{Statistical analysis}

A paired Student's $t$-test was used to compare $T_{0}$ and $\mathrm{T}_{5 \mathrm{~min}}$ plasma haemolysis and ci-miRNAs and $\mathrm{T}_{0}$ and $\mathrm{T}_{4 \mathrm{~h}}$ muscle miRNA abundance. A Shapiro-Wilk test was performed to confirm that the $C_{\mathrm{t}}$ data was normally distributed and the Benjamini and Hochberg method used to correct for multiple testing. Pearson's rank correlation was used to evaluate correlation between $T_{0}$ and $T_{5 \min }$ ci-miRNA expression level and absorbance at $414 \mathrm{~nm}$, and a paired Student's $t$-test used to assess the significance of that correlation. Significance was set at $P \leq 0.05$.

\section{Prediction and annotation of miRNA targets}

Prediction of potential target genes and pathway analysis was completed using the DIANA-mirPath software [30] in the Diana Tools suite. The DIANA-microT-CDS algorithm was used to predict potential target genes for $\mathrm{DE}$ miRNAs; these were then utilised by miRPath for KEGG pathway analysis, enriched through analysis of all predicted targets. A 0.8 default setting was used as the MicroT prediction score threshold with species set to human since there was no equine option. Since there is a high conservation of miRNAs accross species suggesting the sharing of biological functions, it was believed that inferences could be made on the function of equine miRNAs based on their human homologues. Significance for over-represented KEGG pathways was set as $P \leq 0.05$ following Benjamini and Hochberg correction for multiple testing.

\section{Results}

Quality control for miRNA quantification

The miRNA data for $7 / 20$ horses from cohort A failed quality control and were omitted from analysis. All samples from cohort B passed quality control.

\section{Physiological exercise parameters}

For all horses undertaking an exercise test in the study, the average $V_{\text {peak }}$ was $16.9 \pm 0.98 \mathrm{~m} / \mathrm{s}$ (range 15.2-18.5), the average $\mathrm{HR}_{\text {peak }} 221.4 \pm 7.1 \mathrm{bpm}$ (range 209-232) and the average total exercise distance $1130.19 \pm 145.1 \mathrm{~m} / \mathrm{s}$ (range 876-1471). The average pre- and 5 mins postexercise plasma [LA] were $0.46 \pm 0.09 \mathrm{mmol} / \mathrm{l}$ (range $0.33-0.63$ ) and $26.3 \pm 3.68 \mathrm{mmol} / \mathrm{l}$ (range 19.2-32.1), respectively. There were no significant differences between males and females regarding any of the exercise and physiological measurements.

\section{Cohort A: Plasma ci-miRNA}

In the equine plasma samples, $29 \%(52 / 179)$ of the miRNAs on the human plasma panel were detected at both timepoints, all of which had increased expression (3.23 \pm 1.5 fold mean increase, 1.1-10.3 range increase) at $\mathrm{T}_{5 \mathrm{~min}}$ when compared to $\mathrm{T}_{0}$. However, absorbance $>0.2 \mathrm{AU}$ at $414 \mathrm{~nm}$ were detected in $11 / 13 \mathrm{~T}_{0}$ and all $\mathrm{T}_{5 \mathrm{~min}}$ plasma samples for horses in cohort $\mathrm{A}$, with absorbance peaks significantly higher in the $\mathrm{T}_{5 \min }$ samples $\left(P=1.2^{-05}\right.$; Fig. 1$)$. The $\mathrm{T}_{0}$ absorbance ranged from 


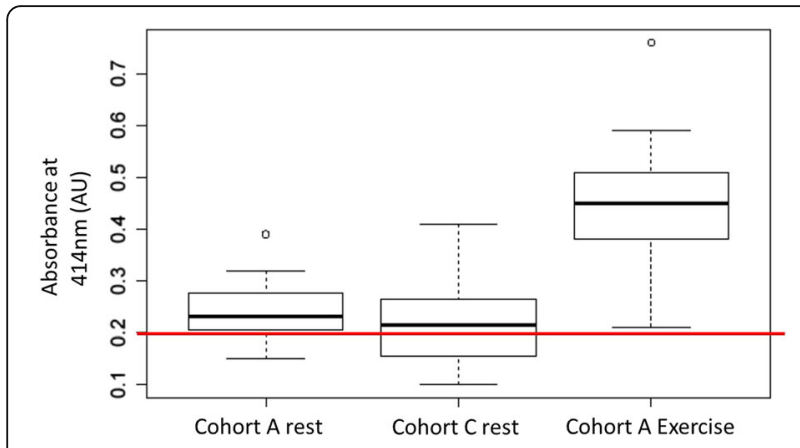

Fig. 1 Box and whiskers plot depicting the degree of haemolysis based on absorbance at $414 \mathrm{~nm}$ for $n=13$ equine plasma samples before and after exercise (cohort $A$ ) and from $n=12$ equine plasma sampes from horses at rest (cohort C). A value of $\leq 0.2$ absorption units was used as the threshold for acceptable haemolysis and accurate quantification of ci-miRNA detection. The red line indicates the threshold above which accurate quantification of ci-miRNA is not possible

0.15-0.39 AU with an average of $0.25 \pm 0.06 \mathrm{AU}$ while the $\mathrm{T}_{5 \mathrm{~min}}$ values ranged from $0.26-0.76 \mathrm{AU}$ with an average of $0.46 \pm 0.13$ AU. The expression levels of 35 miRNAs were significantly correlated $(r>0.55, P<0.05)$ with absorbance and thus haemolysis levels in both $\mathrm{T}_{0}$ and $\mathrm{T}_{5 \mathrm{~min}}$ samples while 13 miRNA expression levels were only significantly correlated with haemolysis in $\mathrm{T}_{0}$ samples; 4 miRNAs were not correlated with haemolysis at either time point. Due to the high levels of haemolysis in the plasma samples and the strong positive correlation between haemolysis and miRNA abundance (Fig. 2), it was not possible to accurately quantify exercise-induced changes in plasma ci-miRNA expression in the present study. There were no significant differences between males and females regarding any of the haemolysis and ci-miRNA measurements.

\section{Cohort B: Skeletal muscle miRNA}

In the equine skeletal muscle biopsy samples, 55\% (98/ 179) of the miRNAs on the human plasma panel were detected at both $\mathrm{T}_{0}$ and $\mathrm{T}_{4 \mathrm{~h}}$. Following the Benjamini and Hochberg correction for multiple testing, 5 of these miRNAs were significantly $\mathrm{DE}$ at $\mathrm{T}_{4 \mathrm{~h}}(P<0.05$; Fig. 3$)$. The greatest expression difference was detected in miRNA miR-21-5p which increased $0.92 \pm 0.2$ fold. The expression of the miR-30b-5p and miR-30e-5p family members increased $0.52 \pm 0.1$ and $0.64 \pm 0.2$ fold, respectively. Expression of let-7d-3p and let-7d-5p decreased $0.68 \pm 0.1$ and $0.44 \pm 0.1$ fold, respectively. Using the DIANA-microT-CDS algorithm a total of 1687 gene targets for these 5 miRNAs were identified. The largest number of gene targets were identified for the miR-30 family members. In total 1077 of the predicted targets were unique to these 2 miRNAs while only 3 gene targets were predicted for let-7d-3p. The most overrepresented KEGG pathways for each miRNA based on a predicted target gene list are summarised in Table 1 $[31,32]$. Pathways previously implicated in the equine exercise response were significantly enriched among predicted targets of the DE miRNAs (Table 2). These included the Jak-STAT signaling, MAPK signaling, insulin signaling and mTOR signaling pathways and long-term potentiation.

\section{Cohort C: Degree of plasma haemolysis}

The average absorbance at $414 \mathrm{~nm}$ for this cohort was $0.21 \pm 0.09 \mathrm{AU}$, ranging from 0.10-0.41 AU (Fig. 1); $7 / 12$ samples had absorbtion peaks >0.2 AU. There was no significant difference in resting haemolysis levels between cohorts $\mathrm{A}$ and $\mathrm{C}$, confirming that

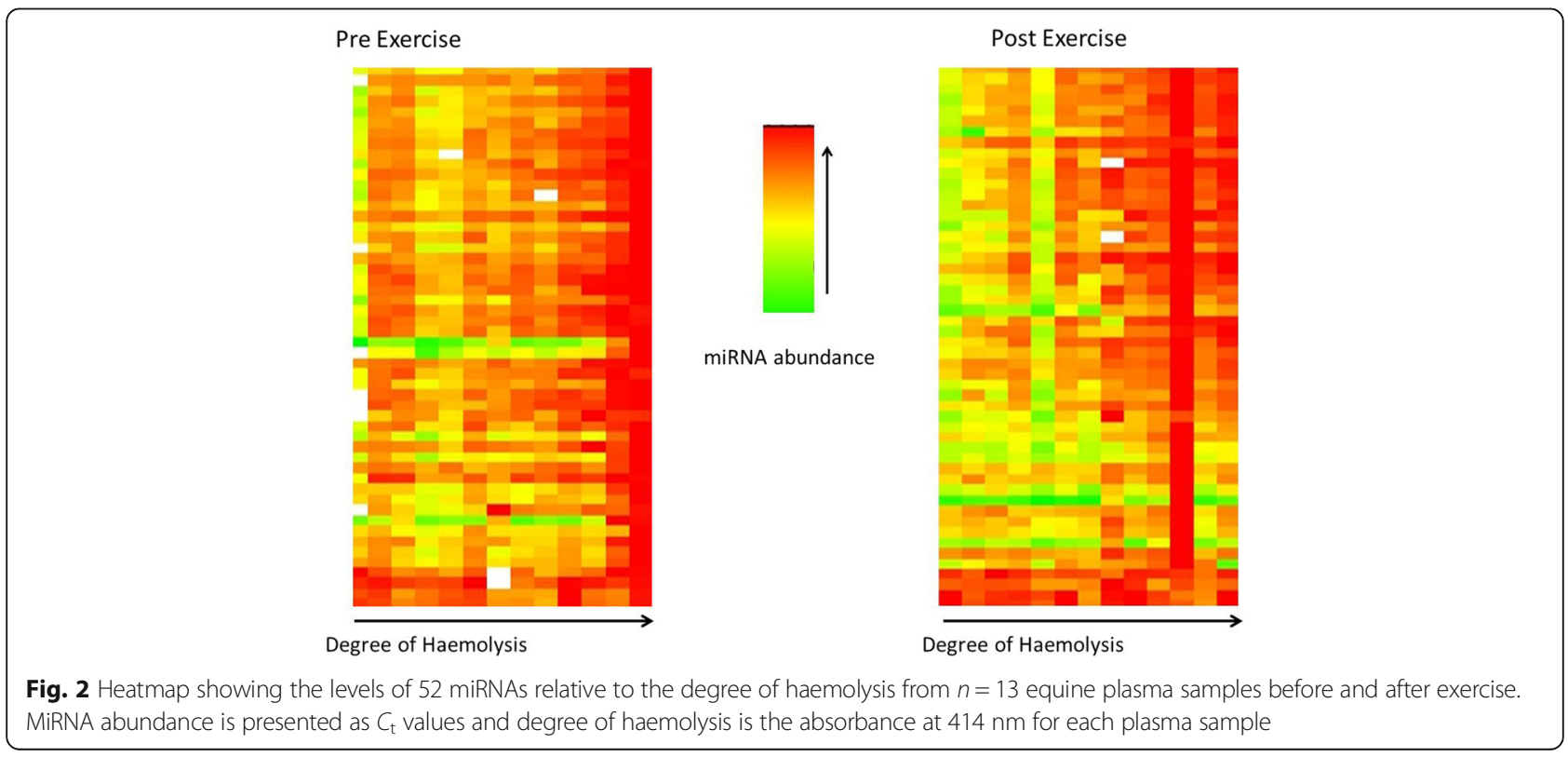




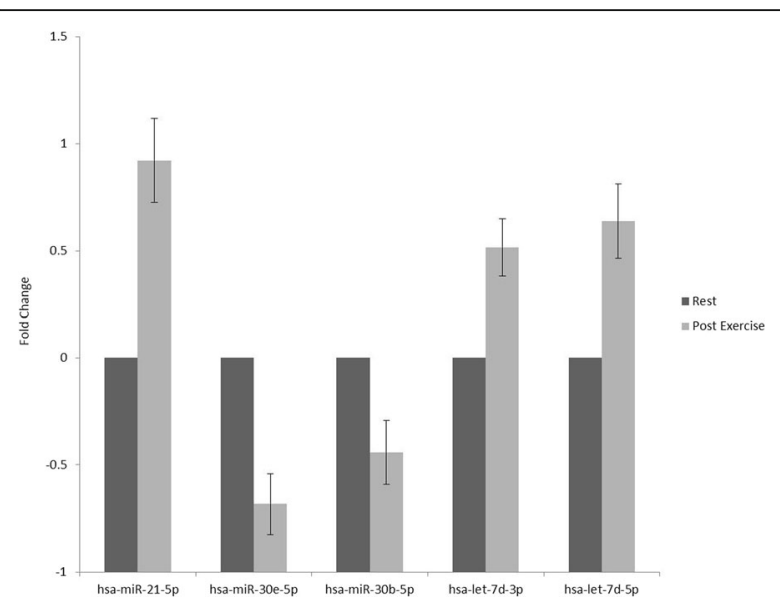

Fig. 3 Real time GRT-PCR results for miRNAs differentially expressed in skeletal muscle before and following exercise from $n=11$ Thoroughbred horses. The standard $2-\Delta \Delta C T$ method was used to determine mean fold changes in gene expression. All Ct values were normalised using the global normalisation function in the GeneEx software package. A Student's t-test was used to identify significant differences in mRNA abundance between time-points

haemolysis at rest is prevelant in $\mathrm{Tb}$ racehorses in high-intensity exercise training.

\section{Discussion}

The stability and ease of access for collecting samples for ci-miRNA measurement make them attractive as potential diagnostic biomarkers. However, in order to investigate ci-miRNAs as diagnostic tools it is vital to identify normal patterns of expression for different scenarios including responses to exercise. Furthermore, rigourous quality control measures must be in place to ensure reliable results. One adverse situation identified to cause variation in plasma ci-miRNA measurement is RBC haemolysis resulting in leakage of miRNAs into the plasma [22, 23, 33]. This is important to consider since there appears to be distinct miRNA profiles for the various cell types in blood [22]. Keeping this in mind, the use of stringent quality controls and careful sample handling has resulted in the accurate quantification of resting and post-exercise plasma ci-miRNAs from human subjects [12].

In the present study, haemolysis above the threshold for accurate quantification of ci-miRNAs was identified in the majority of the resting plasma samples as well as in all of the $\mathrm{T}_{5 \min }$ plasma samples. Intravascular haemolysis has been identified to occur in horses following racing [26], exercise on the treadmill [34-36] and after work on the gallops [27]. Exercise-induced haemolysis in horses primarily results from increased fragility of erythrocytes [37, 38], hypothesized to be due to the frequent accumulation of RBCs in the spleen [35, 37]. Changes in $\mathrm{pH}$ due to anaerobic exercise has also been associated with increased erythrocyte fragility [38], which also occurs in human althletes following maximal exercise [39]. It was interesting to note that in the present study even resting plasma samples had a degree of haemolysis above the threshold for accurate quantification of ci-miRNA detection [22]. We hypothesised that since the horses were in active training for 6 days of the week, which is typical for a flat racehorse training establishment, this resulted in almost daily and consistent exercise-induced insult to the RBCs resulting in persistent levels of haemolysis. Thus, the degree of plasma haemolysis and subsequent effect on miRNA abundance is important to consider when investigating plasma ci-miRNA levels in horses that are in active exercise programmes, even when evaluating pre-exercise samples.

In the present study plasma samples were analysed spectrophotometrically to determine levels of free $\mathrm{Hb}$, as this is an extremely simple and accurate method to determine the extent of plasma haemolysis [22]. For the 48/52 miRNAs detected in plasma in the current study, miRNA abundance was significantly correlated with absorbance at one or both sampling time points supporting correlation between haemolysis and plasma ci-miRNA detection. Since measurement of $\geq 90 \%$ of the detected plasma ci-miRNAs in the current study was correlated with haemolysis, an accurate assessment of the changes in ci-miRNA abundance in response to exercise could not be made. It is believed, however, that this problem could be overcome through the quantification of exosomal ci-miRNAs (plasma miRNAs packaged in vesicles) rather than measurement of all plasma ci-miRNAs. This technique would exclude all unpackaged miRNAs which may have been leaked into the plasma from haemolysed erythrocytes.

Our research group previously demonstrated that mRNA in equine skeletal muscle responds to a single

Table 1 The most over-represented KEGG pathways for each miRNA based on a predicted target gene list

\begin{tabular}{llllll}
\hline miRNA & Fold change & adjusted $P$ value & $n$ genes & Top KEGG pathway & $P$ value \\
\hline hsa-let-7d-3p & -0.68 & 0.002 & 3 & NA & NA \\
hsa-miR-21-5p & 0.92 & 0.002 & 243 & Cytokine-cytokine receptor interaction & $2.48 E-06$ \\
hsa-let-7d-5p & -0.44 & 0.022 & 370 & ECM-receptor interaction & $9.22 E-06$ \\
hsa-miR-30b-5p & 0.52 & 0.023 & 1113 & Ubiquitin mediated proteolysis & $4.76 E-10$ \\
hsa-miR-30e-5p & 0.64 & 0.042 & 1153 & Ubiquitin mediated proteolysis & 2.92E-08 \\
\hline
\end{tabular}


Table 2 Pathways previously implicated in the equine exercise response that were significantly enriched among predicted targets of the differntially expressed miRNAs

\begin{tabular}{llll}
\hline KEGG pathway & $P$ value & $n$ genes & $n$ miRNAs \\
\hline B cell receptor signalling pathway & $1.4637 \mathrm{E}-06$ & 16 & 4 \\
Ubiquitin mediated proteolysis & $1.4637 \mathrm{E}-06$ & 25 & 4 \\
Neurotrophin signalling pathway & $3.75787 \mathrm{E}-06$ & 22 & 4 \\
T cell receptor signalling pathway & $3.75787 \mathrm{E}-06$ & 20 & 4 \\
TGF-beta signalling pathway & $9.00943 \mathrm{E}-06$ & 15 & 4 \\
PI3K-Akt signalling pathway & $4.61509 \mathrm{E}-05$ & 43 & 4 \\
Long-term potentiation & 0.000171436 & 13 & 4 \\
Regulation of actin cytoskeleton & 0.000171436 & 28 & 4 \\
MAPK signalling pathway & 0.000293552 & 34 & 4 \\
Jak-STAT signalling pathway & 0.000485158 & 22 & 4 \\
Axon guidance & 0.000671812 & 22 & 3 \\
Arrhythmogenic right ventricular & 0.000834821 & 13 & 3 \\
cardiomyopathy & & & \\
Hepatitis B & 0.000838527 & 21 & 4 \\
Transcriptional misregulation & 0.002038503 & 26 & 4 \\
in cancer & & & \\
Hypertrophic cardiomyopathy & 0.002113538 & 13 & 3 \\
Insulin signalling pathway & 0.002113538 & 19 & 4 \\
Mucin type O-Glycan biosynthesis & 0.002113538 & 6 & 3 \\
Prostate cancer & 0.0021797 & 13 & 4 \\
mTOR signalling pathway & 0.002349162 & 11 & 4 \\
Acute myeloid leukaemia & 0.003086604 & 9 & 4 \\
Adherens junction & 0.004193219 & 14 & 4 \\
\hline & & & \\
\hline
\end{tabular}

bout of exercise as well as exercise training [31, 32]. These results underpinned our interest in evaluating the effect that exercise may have on equine skeletal muscle miRNA expression. The initial profiling for equine skeletal muscle miRNA expression in the present study was carried out on horses just entering sprint training. This was based on results from previous studies in our laboratory in which $\mathrm{Tb}$ skeletal muscle was found to have the greatest transcriptional response to intense exercise at this training stage [31]. The $4 \mathrm{~h}$ post-exercise sampling time was also chosen based on previous work performed by our research group where it was identified to be the time-point exhibiting the most transcriptional activity [31]. These observations are in agreement with several human exercise studies [40-42].

In this study we observed changes in equine skeletal muscle miRNA abundance following exercise. All five of the DE miRNAs from skeletal muscle expressed at $T_{4}$ in the present study had been identified amongst the 48 miRNAs detected from haemolysed plasma samples from horses in cohort A. Furthermore, all five are known equine miRNAs with miR-30e-5p previously reported to be expresesed in muscle in a tissue-specific manner [20].
Of great interest is that these five DE miRNAs are amongst $167 \mathrm{DE}$ miRNAs reported from pre- and $30 \mathrm{~min}$ post-endurance exercise whole-blood samples in a group of $n=14$ pure-breed/half-breed Arabian horses [21].

In the present study, two members of the let-7 family of miRNAs, let-7d-3p and let-7d-5p, and miR-21-5p had increased expression following exercise. The Let-7 family of miRNAs are involved in the regulation of glucose homeostasis and insulin sensitivity [43], both key processes in energy metabolism during exercise. Mir- 21 has been shown to be involved in the fibrogenic pathway and the progression of Duchenne muscular dystrophy [44], so may play a role in muscle remodelling during exercise training.

Two members of the miR-30 family, miR-30b-5p and miR-30e-5p, had decreased expression post-exercise. The miR-30 family has been shown to be downregulated in $m d x 4 c v$ mice (models for Duchenne muscular dystrophy), with in vitro analysis indicating that miR-30 miRNAs are decreased following injury and are increased during myoblast differentiation [45]. These findings suggest that these miRNAs may play an important role in skeletal muscle growth and repair [45]. The decreased expression in response to exercise may be related to repair of exercise-induced muscle damage. The over-representation of miRNA gene targets in exercise-related KEGG pathways certainly suggests that miRNAs play a key role in the modulation of gene expression in response to exercise.

\section{Conclusions}

In conclusion, our results indicate even resting plasma from horses in high-intensity training may not be suitable for ci-miRNA quantification due to haemolysis, although it is possible this problem could be overcome through the quantification of exosomal ci-miRNAs. However, identification of DE miRNAs in skeletal muscle indicates that modification of miRNA expression may contribute to adaptive training responses in Tbs. It is likely that additional miRNAs are differentially regulated in response to exercise but were undetectable due to the use of a human plasma panel. Future studies using a global platform such as miRNA sequencing will be required to generate a comprehensive profile of miRNAs in equine skeletal muscle at rest and following exercise. The most powerful approach to such an investigation would be an analysis of the miRNA-Seq transcriptome.

\section{Endnotes}

${ }^{1}$ Polar Equine S810i, Polar Electro Ltd., Warwick, UK

${ }^{2}$ GPSports Systems SPI10, Canberra, Australia

${ }^{3}$ YSI 2300 stat plus, YSI UK Ltd., Hampshire, UK 


\author{
${ }^{4}$ biofluids miRCURYTM RNA Isolation Kit, Exiqon, \\ Denmark \\ ${ }^{5}$ miRCURY LNA ${ }^{\text {tu }}$ Universal RT microRNA PCR, poly- \\ adenylation and cDNA synthesis kit, Exiqon, Denmark \\ ${ }^{6}$ NanoDrop ${ }^{\text {tw }}$ Bioanalyzer ND2000, Labtech, Uckfield, UK \\ ${ }^{7}$ Jørgen KRUUSE A/S, Langeskov, Denmark \\ ${ }^{8}$ Ambion, ThermoFisher Scientific Inc., Darmstadt, Germany \\ ${ }^{9}$ Invitrogen, ThermoFisher Scientific Inc., Darmstadt, \\ Germany \\ ${ }^{10}$ RNase-free DNase, Qiagen Ltd., Manchester, UK \\ ${ }^{11}$ Mini-kit, Qiagen Ltd., Manchester, UK. \\ ${ }^{12}$ Agilent Technologies Ireland, Ltd.
}

\section{Abbreviations}

[Hb]: Haemoglobin concentration; [LA]: Lactate concentrations;

AU: Absorption units; ci-miRNAs: Circulating miRNAs; $C_{\mathrm{t}}$ : Cycle threshold;

DE: Differentially expressed; EDTA: Ethylenediamine tetraacetic acid; HR: Heart rate; miRNAs: MicroRNAs; mRNA: Messenger ribonucleic acid; RBC: Red blood cells; RT-qPCR: Reverse transcription quantitative realtime polymerase chain reaction; $T_{0}$ : before exercise (rest); $T_{4 h r}: 4 \mathrm{~h}$ after exercise; $T_{5 \min }: 5$ mins after exercise; Tb: Thoroughbreds; WD: Work day

\section{Acknowledgements}

The authors wish to express their appreciation to the trainer Mr. Jim Bolger and his staff.

\section{Funding}

This study has emanated from research conducted with the financial support of Science Foundation Ireland under Grant Number 11/PI/1166.

\section{Availability of data and materials}

All data generated or analysed during this study are included in this published article. Accession numbers for the miRNA data generated in this study are: MIMAT0004484 (hsa-let-7d-3p), MIMAT0000076 (hsa-miR-21-5p), MIMAT0000065 (hsalet-7d-5p), MIMAT0000420 (hsa-miR-30b-5p) and MIMAT0000692 (hsa-miR-30e-5p).

\section{Authors' contributions}

(i) BAMCG, EWH and LMK designed the experimental protocol; (ii) BAMCG MEG, KFG, CLMcG and LMK collected all data; (iii) BAMcG, MEG, JAB, EWH and LMK performed all data analysis and interpretation; (iv, v) all authors contributed to the writing, revision and final approval of the manuscript.

\section{Ethics approval}

University College Dublin Animal Research Ethics Committee approval, a Department of Health License (B100/3525) and explicit owner/trainer informed consent were obtained for the use of the horses and procedures in this study.

\section{Consent for publication}

Not applicable.

\section{Competing interests}

None of the authors has any financial or personal relationships that could inappropriately influence or bias the content of the paper. E.W.H. is a shareholder in Plusvital Ltd., an equine nutrition and genetic testing company. Plusvital Ltd. has been granted a license for commercial use of data contained within patent applications: United States Provisional Serial Number 61/136553 and Irish patent application number 2008/0735, Patent Cooperation Treaty filing: A method for predicting athletic performance potential, September 7, 2009. E.W.H. and L.M.K. are named on the applications. The patent contents are not related to this manuscript. Plusvital Ltd. had no part in the research in the manuscript.

\section{Publisher's Note}

Springer Nature remains neutral with regard to jurisdictional claims in published maps and institutional affiliations.

\section{Author details}

UCD School of Agriculture and Food Science, Section of Animal \& Crop Sciences, University College Dublin, Belfield, Dublin 4, Ireland. ${ }^{2}$ UCD School of Veterinary Medicine, Section of Veterinary Biomedical Sciences, University College Dublin, Belfield, Dublin 4, Ireland.

Received: 17 November 2016 Accepted: 16 November 2017

Published online: 22 November 2017

\section{References}

1. Bartel DP. MicroRNAs: genomics, biogenesis, mechanism, and function. Cell. 2004;116:281-97.

2. Cai $Y$, Xiaomin $Y$, Songnian $H, Y u$ JA. Brief review on the mechanisms of miRNA regulation. Genomics Proteomics Bioinformatics. 2009;7:147-54.

3. Cortez MA, Calin GA. MicroRNA identification in plasma and serum: a new tool to diagnose and monitor diseases. Expert Opin Biol Ther. 2009;9:703-11.

4. Chen X, Ba Y, Ma L, Cai X, Yin Y, Wang K, Guo J, Zhang Y, Chen J, Guo X, Li Q, Li X, Wang W, Zhang Y, Wang J, Jiang X, Xiang Y, Xu C, Zheng P, Zhang J, Li R, Zhang H, Shang X, Gong T, Ning G, Wang J, Zen K, Zhang J, Zhang C-Y. Characterization of microRNAs in serum: a novel class of biomarkers for diagnosis of cancer and other diseases. Cell Res. 2008;18:997-1006.

5. Vickers KC, Palmisano BT, Shoucri BM, Shamburek RD, Remaley AT. MicroRNAs are transported in plasma and delivered to recipient cells by high-density lipoproteins. Nat Cell Biol. 2011:13:423-33.

6. Turchinovich A, Weiz L, Langheinz A, Burwinkel B. Characterization of extracellular circulating microRNA. Nucleic Acids Res. 2011:39:7223-33.

7. Mitchell PS, Parkin RK, Kroh EM, Fritz BR, Wyman SK, Pogosova-Agadjanyan EL, Peterson A, Noteboom J, O'Briant KC, Allen A, Lin DW. Circulating microRNAs as stable blood-based markers for cancer detection. Proc Natl Acad Sci. 2008; 105:10513-8.

8. Reid G, Kirschner MB, van Zandwijk N. Circulating microRNAs: association with disease and potential use as biomarkers. Crit Rev Oncol Hematol. 2011; 80:193-208.

9. Baggish AL, Hale A, Weiner RB, Lewis GD, Systrom D, Wang F, Wang TJ, Chan SY. Dynamic regulation of circulating microRNAs during acute exhaustive exercise and sustained aerobic exercise training. J Physiol. 2011:589:3983-94

10. Bye A, Rosjo H, Aspenes ST, Condorelli G, Omland T, Wisloff U. Circulating microRNAs and aerobic fitness-the HUNT-study. PLoS One. 2013:8:e57496.

11. Russell AP, Lamon S, Boon H, Wada S, Guller I, Brown EL, Chibalin AV, Zierath JR, Snow RJ, Stepto N, Wadley GD, Akimoto T. Regulation of miRNAs in human skeletal muscle following acute endurance exercise and short-term endurance training. J Physiol. 2013;591:4637-53.

12. Nielsen S, Akerstrom T, Rinnov A, Yfanti C, Scheele C, Pedersen BK, Laye MJ. The miRNA plasma signature in response to acute aerobic exercise and endurance training. PLoS One. 2014;9:e87308. doi:10.1371/journal.pone. 0087308 .

13. Davidsen PK, Gallagher IJ, Hartman JW, Tarnopolsky MA, Dela F, Helge JW, Timmons JA, Phillips SM. High responders to resistance exercise training demonstrate differential regulation of skeletal muscle microRNA expression. J Appl Physiol. 2011;110:309-17.

14. Keller P, Vollaard NB, Gustafsson T, Gallagher IJ, Sundberg CJ, Rankinen T, Britton SL, Bouchard C, Koch LG, Timmons JA. A transcriptional map of the impact of endurance exercise training on skeletal muscle phenotype. J Appl Physiol. 2011;110:46-59.

15. Guescini M, Guidolin D, Vallorani L, Casadei L, Gioacchini AM, Tibollo P, Battistelli M, Falcieri E, Battistin L, Agnati LF, Stocchi V. C2C12 myoblasts release micro-vesicles containing mtDNA and proteins involved in signal transduction. Exp Cell Res. 2010;316:1977-2984.

16. Pedersen BK, Febbraio MA. Muscles, exercise and obesity: skeletal muscle as a secretory organ. Nat Rev Endocrinol. 2012:8:457-65.

17. Van der Kolk JH, Pacholewska A, Gerber V. The role of microRNAs in equine medicine: a review Vet $Q$. 2015:35:88-96.

18. Barrey E, Bonnamy B, Barrey EJ, Mata X, Chaffaux S, Guerin G. Muscular microRNA expressions in healthy and myopathic horses suffering from polysaccharide storage myopathy or recurrent exertional rhabdomyolysis. Equine Vet J. 2010:42:303-10.

19. Gim JA, Ayarpadikannan S, Eo J, Kwon YJ, Choi Y, Lee HK, Park KD, Yang YM, Cho BW, Kim HS. Transcriptional expression changes of glucose metabolism genes after exercise in thoroughbred horses. Gene. 2014;547:152-8. 
20. Kim MC, Lee SW, Ryu DY, Cui FJ, Bhak J, Kim Y. Identification and characterization of microRNAs in normal equine tissues by next generation sequencing. PLoS One. 2014;9:e93662

21. Mach N, Plancade S, Pacholewska A, Lecardonnel J, Riviere J, Moroldo M, Vaiman A, Morgenthaler C, Beinat M, Nevot A, Robert C, Barrey E. Integrated mRNA and miRNA expression profiling in blood reveals candiate biomarkers associated with endurance exercise in the horse. Sci Rep. 2016;6:22932.

22. Kirschner MB, Kao SC, Edelman JJ, Armstrong NJ, Vallely MP, van Zandwijk $\mathrm{N}$, Reid G. Haemolysis during sample preparation alters microRNA content of plasma. PLoS One. 2011;6:e24145.

23. McDonald JS, Milosevic D, Reddi HV, Grebe SK, Algeciras-Schimnich A. Analysis of circulating microRNA: preanalytical and analytical challenges. Clin Chem. 2011:57:833-40.

24. Blondal T, Jensby Nielsen S, Baker A, Andreasen D, Mouritzen P, Wrang Teilum M, Dahlsveen IK. Assessing sample and miRNA profile quality in serum and plasma or other biofluids. Methods. 2013;59:S1-6.

25. Kirschner MB, Edelman JJ, Kao SC, Vallely MP, van Zandwijk N, Reid G. The impact of hemolysis on cell-free microRNA biomarkers. Front Genet. 2013;4:94.

26. Masini AP, Tedeschi D, Badagli P, Sighieri C, Lubas C. Exercise-induced intravascular haemolysis in standardbred horses. Comp Clin Path. 2003;12: 45-8.

27. Cywinska A, Szarska E, Kowalska A, Ostaszewski P, Schollenberger A. Gender differences in exercise-induced intravascular haemolysis during race training in thoroughbred horses. Res Vet Sci. 2011;90:133-7.

28. Fonseca RG, Kenny DA, Hill EW, Katz LM. The association of various speed indices to training responses in thoroughbred flat racehorses measured with a global positioning and heart rate monitoring system. Equine Vet J. 2010;42:51-7.

29. Dingboom EG, Dijkstra G, Enzerink E, van Oudheusden HC, Weijs WA. Postnatal muscle fibre composition of the gluteus medius muscle of Dutch Warmblood foals; maturation and the influence of exercise. Equine Vet J. 1999;31:95-100

30. Vlachos IS, Kostoulas N, Vergoulis T, Georgakilas G, Reczko M, Maragkakis M, Paraskevopoulou MD, Prionidis K, Dalamagas T, Hatzigeorgiou AGDIANA. miRPath v.2.0: investigating the combinatorial effect of microRNAs in pathways. Nucleic Acids Res. 2012:40:W498-504

31. McGivney BA, Eivers SS, MacHugh DE, MacLeod JN, O'Gorman GM, Park SD, Katz LM, Hill EW. Transcriptional adaptations following exercise in thoroughbred horse skeletal muscle highlights molecular mechanisms that lead to muscle hypertrophy. BMC Genomics. 2009;10:638.

32. McGivney BA, McGettigan PA, Browne JA, Evans AC, Fonseca RG, Loftus BJ, Lohan A, MacHugh DE, Murphy BA, Katz LM, Hill EW. Characterization of the equine skeletal muscle transcriptome identifies novel functional responses to exercise training. BMC Genomics. 2010;11:398.

33. Pritchard CC, Kroh E, Wood B, Arroyo JD, Dougherty KJ, Miyaji MM, Tait JF, Tewari M. Blood cell origin of circulating microRNAs: a cautionary note for cancer biomarker studies. Cancer Prev Res. 2012;5:492-7.

34. Schott HC, Hodgson DR, Bayly WM. Haematuria, pigmenturia and proteinuria in exercising horses. Equine Vet J. 1995;27:67-72.

35. Hanzawa K, Higara A, Yoshida Y, Hara H, Kai M, Kubo K, Watanabe S. Effects of exercise on plasma haptoglobin composition in control and splenectomized thoroughbred horses. J Equine Sci. 2002;13:89-92.

36. Inoue $Y$, Matsui A, Asai $Y$, Aoki F, Matsui T, Yano H. Effect of exercise on iron metabolism in horses. Biol Trace Elem Res. 2005;107:33-42.

37. Hanzawa K, Kai M, Hirag A, Watanabe S. Fragility of red cells during exercise is affected by blood pH and temperature. Equine Vet J. 1999;31:610-1.

38. Hanzawa K, Watanabe S. Changes in osmotic fragility of erythrocytes during exercise in athletic horses. J Equine Sci. 2000;11:51-61.

39. Smith JA, Telford RD, Kolbuch-Braddon M, Weidermann MJ. Lactate/H+ uptake by red blood cells during exercise alters their physical properties. Eur Appl Physiol Occup Physiol. 1995;75:54-61.

40. Yang Y, Creer A, Jemiolo B, Trappe S. Time course of myogenic and metabolic gene expression in response to acute exercise in human skeletal muscle. J Appl Physiol. 2005;98:1745-52.

41. Mahoney DJ, Safdar A, Parise G, Melov S, Fu M, MacNeil L, Kaczor J, Payne ET, Tarnopolsky MA. Gene expression profiling in human skeletal muscle during recovery from eccentric exercise. Am J Physiol Regul Integr Comp Physiol. 2008;294:R1901-10.

42. Nader GA, von Walden F, Liu C, Lindvall J, Gutmann L, Pistilli EE, Gordon PM. Resistance exercise training modulates acute gene expression during human skeletal muscle hypertrophy. J Appl Physiol. 2014;116:693-702.
43. Frost RJ, Olson EN. Control of glucose homeostasis and insulin sensitivity by the Let-7 family of microRNAs. Proc Natl Acad Sci. 2011:108:21075-80.

44. Ardite E, Perdiguero E, Vidal B, Gutarra S, Serrano AL, Munoz-Canoves P. PAI1-regulated miR-21 defines a novel age-associated fibrogenic pathway in muscular dystrophy. J Cell Biol. 2012;196:163-75.

45. Guess MG, Barthel KK, Harrison BC, Leinwand LA. miR-30 family microRNAs regulate myogenic differentiation and provide negative feedback on the microRNA pathway. PLoS One. 2015;10:e0118229.

\section{Submit your next manuscript to BioMed Central and we will help you at every step:}

- We accept pre-submission inquiries

- Our selector tool helps you to find the most relevant journal

- We provide round the clock customer support

- Convenient online submission

- Thorough peer review

- Inclusion in PubMed and all major indexing services

- Maximum visibility for your research

Submit your manuscript at www.biomedcentral.com/submit
Biomed Central 